\title{
On the Recursive Sequence
}

$$
x_{n+1}=\frac{x_{n-29}}{1+x_{n-4} x_{n-9} x_{n-14} x_{n-19} x_{n-24}}
$$

Burak Oğul ${ }^{1 *}$, Dağıstan Şimşek ${ }^{2}$

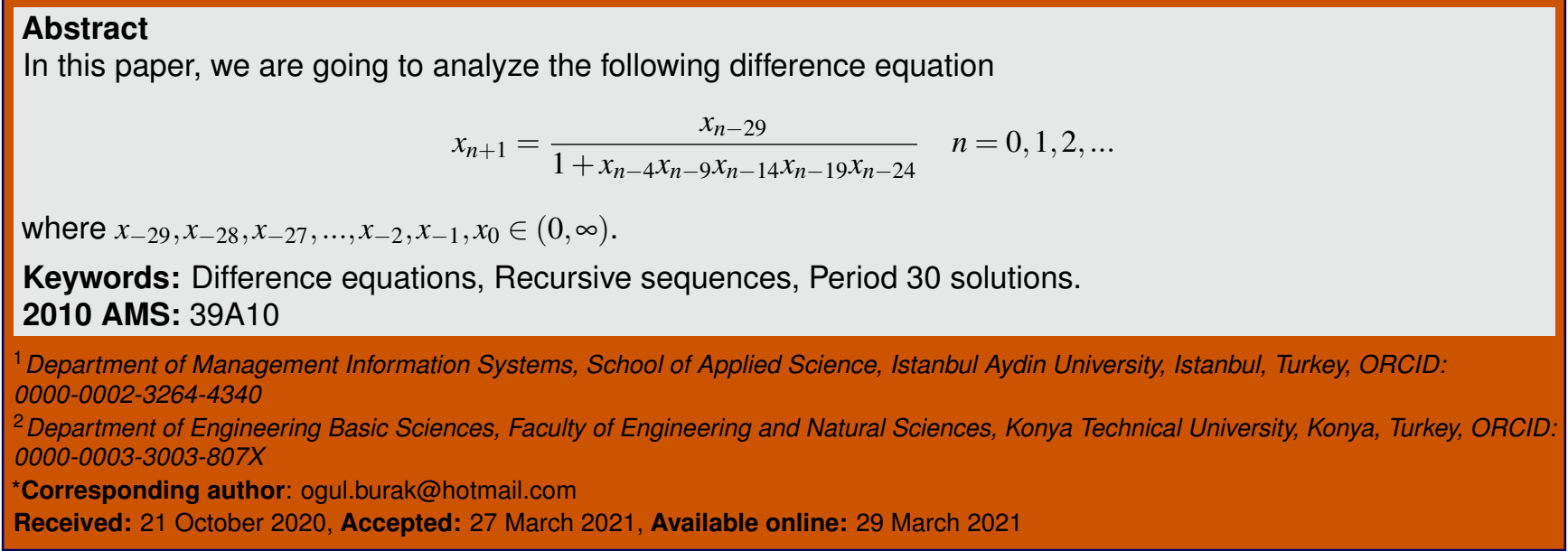

\section{Introduction}

Difference equation is a very diverse field that is effective in almost every branch of applied mathematics. Recently, researchers have shown great interest in studying the behavior of solutions of nonlinear difference equations. Difference equations are used in many fields such as population biology, economics, probability theory, genetics, psychology, mathematical modeling. There are many articles on difference equations, for example; [24]-[28]

Cinar, studied the following problem with positive initial values:

$$
x_{n+1}=\frac{x_{n-1}}{-1+a x_{n} x_{n-1}},
$$

for $n=0,1,2, \ldots$ in [2] respectively.

Simsek et. al., studied the following problems with positive initial values,

$$
\begin{aligned}
& x_{n+1}=\frac{x_{n-3}}{1+x_{n-1}} \\
& x_{n+1}=\frac{x_{n-5}}{1+x_{n-2}}, \\
& x_{n+1}=\frac{x_{n-5}}{1+x_{n-1} x_{n-3}}
\end{aligned}
$$


for $n=0,1,2, \ldots$ in [5]-[7] respectively.

Elsayed studied the behavior of the solution of the following difference equation,

$$
x_{n+1}=a x_{n-1}+\frac{b x_{n} x_{n-1}}{c x_{n} d x_{n-2}}, \quad n=0,1, \ldots
$$

where the initial conditions $x_{-2} x_{-1}, x_{0}$ are arbitrary positive real numbers and $a, b, c, d$ are positive constants. [15]

Devault et. al. studied the following problems

$$
x_{n+1}=\frac{A}{x_{n}}+\frac{1}{x_{n-2}}
$$

for $n=0,1,2, \ldots$ in [23] and showed every positive solution of the equation where $A \in(0, \infty)$.

Stevic et. al. studied on a product-type system of difference equations of second order solvable in closed form in [28]. Shown that the following system of difference equations

$$
z_{n+1}=\frac{z_{n}^{a}}{w_{n-1}^{b}}, w_{n+1}=\frac{w_{n}^{c}}{z_{n-1}^{d}}, n \in \mathbb{N}_{0}
$$

where $a, b, c, d \in \mathbb{Z}, z_{-1}, z_{0}, w_{-1}, w_{0} \in \mathbb{C}$ is solvable in closed form.

In this work, the following non-linear difference equation was studied

$$
x_{n+1}=\frac{x_{n-29}}{1+x_{n-4} x_{n-9} x_{n-14} x_{n-19} x_{n-24}}
$$

where $x_{-29}, x_{-28}, \ldots, x_{-1}, x_{0} \in(0, \infty)$.

\section{Main Results}

Let $\bar{x}$ be the unique positive equilibrium of the 1.1, then clearly,

$$
\bar{x}=\frac{\bar{x}}{1+\overline{x x x x x}} \Rightarrow \bar{x}+\bar{x}^{6}=\bar{x} \Rightarrow \bar{x}^{6}=0 \Rightarrow \bar{x}=0,
$$

so $\bar{x}=0$ can be obtained. For any $k \geq 0$ and $m>k$ notation $i=\overline{k, m}$ means $i=k, k+1, \ldots, m$

Theorem 2.1. Consider the difference equation 1.1. Then the following statements are true.

a) The sequences $x_{30 n-29}, x_{30 n-28}, \ldots, x_{30 n-1}, x_{30 n}$ are being decreased and

$$
a_{1}, a_{2}, \ldots, a_{29}, a_{30} \geq 0
$$

are existed in such that

$$
\lim _{n \rightarrow \infty} x_{30 n-29+k}=a_{1+k}, \quad k=\overline{0,29} .
$$

b)

$$
\prod_{k=0}^{6} \lim _{n \rightarrow \infty} x_{35 n-34-j+5 k}=0, \quad j=\overline{0,4} \quad \text { or } \quad \prod_{k=0}^{6} a_{5 k+i}=0, \quad i=\overline{1,5}
$$

c) $n_{0} \in \mathbb{N}$ such that $x_{n+1} \leq x_{n-24}$ for all $n \geq n_{0}$, then

$$
\lim _{n \rightarrow \infty} x_{n}=0
$$

d) The following formulas below are hold:

$$
x_{30 n+1+k}=x_{-29+k}\left(1-\frac{x_{-4+k} x_{-9+k} x_{-14+k} x_{-19+k} x_{-24+k}}{x_{-4+k} x_{-9+k} x_{-14+k} x_{-19+k} x_{-24+k}} \sum_{j=0}^{6 j} \frac{1}{1+x_{5 i-4+k} x_{5 i-9+k} x_{5 i-14+k} x_{5 i-19+k} x_{5 i-24+k}}\right),
$$




$$
\begin{aligned}
& x_{30 n+6+k}=x_{-24+k}\left(1-\frac{x_{-4+k} x_{-9+k} x_{-14+k} x_{-19+k} x_{-29+k}}{x_{-4+k} x_{-9+k} x_{-14+k} x_{-19+k} x_{-24+k}} \sum_{j=0}^{n} \prod_{i=1}^{6 j+1} \frac{1}{1+x_{5 i-4+k} x_{5 i-9+k} x_{5 i-14+k} x_{5 i-19+k} x_{5 i-24+k}}\right) \\
& x_{30 n+11+k}=x_{-19+k}\left(1-\frac{x_{-4+k} x_{-9+k} x_{-14+k} x_{-24+k} x_{-29+k}}{x_{-4+k} x_{-9+k} x_{-14+k} x_{-19+k} x_{-24+k}} \sum_{j=0}^{6 j+2} \frac{1}{1+x_{5 i-4+k} x_{5 i-9+k} x_{5 i-14+k} x_{5 i-19+k} x_{5 i-24+k}}\right) \\
& x_{30 n+16+k}=x_{-14+k}\left(1-\frac{x_{-4+k} x_{-9+k} x_{-19+k} x_{-24+k} x_{-29+k}}{x_{-4+k} x_{-9+k} x_{-14+k} x_{-19+k} x_{-24+k}} \sum_{j=0}^{6 j+3} \frac{1}{1+x_{5 i-4+k} x_{5 i-9+k} x_{5 i-14+k} x_{5 i-19+k} x_{5 i-24+k}}\right) \\
& x_{30 n+21+k}=x_{-9+k}\left(1-\frac{x_{-4+k} x_{-14+k} x_{-19+k} x_{-24+k} x_{-29+k}}{x_{-4+k} x_{-9+k} x_{-14+k} x_{-19+k} x_{-24+k}} \sum_{j=0}^{n} \prod_{i=1}^{6 j+4} \frac{1}{1+x_{5 i-4+k} x_{5 i-9+k} x_{5 i-14+k} x_{5 i-19+k} x_{5 i-24+k}}\right) \\
& x_{30 n+26+k}=x_{-4+k}\left(1-\frac{x_{-9+k} x_{-14+k} x_{-19+k} x_{-24+k} x_{-29+k}}{x_{-4+k} x_{-9+k} x_{-14+k} x_{-19+k} x_{-24+k}} \sum_{j=0}^{n} \prod_{i=1}^{6 j+5} \frac{1}{1+x_{5 i-4+k} x_{5 i-9+k} x_{5 i-14+k} x_{5 i-19+k} x_{5 i-24+k}}\right) \\
& k=\overline{0,4} \text { holds. }
\end{aligned}
$$

\section{Proof. $\quad$ a) Firstly, from the 1.1}

$$
x_{n+1}=\frac{x_{n-29}}{1+x_{n-4} x_{n-9} x_{n-14} x_{n-19} x_{n-24}}
$$

is obtained. If $x_{n-4} x_{n-9} x_{n-14} x_{n-19} x_{n-24} \in(0,+\infty), \quad$ then $\left(1+x_{n-4} x_{n-9} x_{n-14} x_{n-19} x_{n-24}\right) \in((1,+\infty)$. Since

$$
x_{n+1}<x_{n-29}
$$

$n \in \mathbb{N}$

$$
\lim _{n \rightarrow \infty} x_{30 n-29+k}=a_{1+k}, \quad \text { for } \quad k=\overline{0,29}
$$

existed formulas are obtained.

b) In view of the 1.1 ,

$$
n=30 n \Rightarrow x_{30 n+1}=\frac{x_{30 n-29}}{1+\prod_{k=0}^{5} x_{30 n-29+5 k}}
$$

is obtained. If the limits are put on both sides of the above equality,

$$
\prod_{k=0}^{6} \lim _{n \rightarrow \infty} x_{35 n-34+5 k}=0 \quad \text { or } \quad \prod_{k=0}^{6} a_{5 k+1}=0
$$

is obtained. Similarly for $n=30 n+1, n=30 n+2, n=30 n+3$ and $n=30 n+4$ we can obtain $x_{30 n+2}, x_{30 n+3}, x_{30 n+4}$ and $x_{30 n+5}$. 
c) If there exist $n_{0} \in \mathbb{N}$ such that $x_{n+1} \leq x_{n-24}$ for all $n \geq n_{0}$, then, $a_{1} \leq a_{6} \leq a_{11} \leq a_{16} \leq a_{21} \leq a_{26} \leq a_{1}, \quad a_{2} \leq a_{7} \leq$ $a_{12} \leq a_{17} \leq a_{22} \leq a_{27} \leq a_{2}, \quad a_{3} \leq a_{8} \leq a_{13} \leq a_{18} \leq a_{23} \leq a_{28} \leq a_{3}, \quad a_{4} \leq a_{9} \leq a_{14} \leq a_{19} \leq a_{24} \leq a_{29} \leq a_{4}, \quad a_{5} \leq$ $a_{10} \leq a_{15} \leq a_{20} \leq a_{25} \leq a_{30} \leq a_{5}$. Using (b) we get

$$
\prod_{k=0}^{6} a_{5 k+i}=0, \quad i=\overline{1,5}
$$

Then we see that,

$$
\lim _{n \rightarrow \infty} x_{n}=0
$$

Hence the proof of (c) completed.

d) Subtracting $x_{n-29}$ from the left and right-hand sides in 1.1

$$
x_{n+1}-x_{n-29}=\frac{1}{1+x_{n-4} x_{n-9} x_{n-14} x_{n-19} x_{n-24}}\left(x_{n-4}-x_{n-34}\right)
$$

is obtained and the following formula is produced below, for $n \geq 5$

$$
\begin{aligned}
& x_{5 n-24}-x_{5 n-54}=\left(x_{1}-x_{-29}\right) \prod_{i=1}^{n-5} \frac{1}{1+x_{5 i-4} x_{5 i-9} x_{5 i-14} x_{5 i-19} x_{5 i-24}} \\
& x_{5 n-28}-x_{5 n-53}=\left(x_{2}-x_{-28}\right) \prod_{i=1}^{n-5} \frac{1}{1+x_{5 i-3} x_{5 i-8} x_{5 i-13} x_{5 i-18} x_{5 i-23}} \\
& x_{5 n-27}-x_{5 n-52}=\left(x_{3}-x_{-27}\right) \prod_{i=1}^{n-5} \frac{1}{1+x_{5 i-2} x_{5 i-7} x_{5 i-12} x_{5 i-17} x_{5 i-22}} \\
& x_{5 n-26}-x_{5 n-51}=\left(x_{4}-x_{-26}\right) \prod_{i=1}^{n-5} \frac{1}{1+x_{5 i-1} x_{5 i-6} x_{5 i-11} x_{5 i-16} x_{5 i-21}} \\
& x_{5 n-25}-x_{5 n-50}=\left(x_{5}-x_{-25}\right) \prod_{i=1}^{n-5} \frac{1}{1+x_{5 i} x_{5 i-5} x_{5 i-10} x_{5 i-15} x_{5 i-20}}
\end{aligned}
$$

$6 j$ inserted in 2.1 by replacing $n, j=0$ to $j=n$ is obtained by summing, for $k=\overline{0,4}$

$$
x_{30 n+1+k}-x_{-29+k}=\left(x_{1+k}-x_{-29+k}\right) \sum_{j=0}^{n} \prod_{i=1}^{6 j} \frac{1}{1+x_{5 i-4+k} x_{5 i-9+k} x_{5 i-14+k} x_{5 i-19+k} x_{5 i-24+k}} .
$$

Also, $6 j+1$ inserted in 2.1 by replacing $n, j=0$ to $j=n$ is obtained by summing, for $k=\overline{0,4}$

$$
x_{30 n+6+k}-x_{-24+k}=\left(x_{6+k}-x_{-24+k}\right) \sum_{j=0}^{n} \prod_{i=1}^{6 j+1} \frac{1}{1+x_{5 i-4+k} x_{5 i-9+k} x_{5 i-14+k} x_{5 i-19+k} x_{5 i-24+k}} .
$$

Also, $6 j+2$ inserted in 2.1 by replacing $n, j=0$ to $j=n$ is obtained by summing, for $k=\overline{0,4}$

$$
x_{30 n+11+k}-x_{-19+k}=\left(x_{11+k}-x_{-19+k}\right) \sum_{j=0}^{n} \prod_{i=1}^{6 j+2} \frac{1}{1+x_{5 i-4+k} x_{5 i-9+k} x_{5 i-14+k} x_{5 i-19+k} x_{5 i-24+k}} .
$$

Also, $6 j+3$ inserted in 2.1 by replacing $n, j=0$ to $j=n$ is obtained by summing, for $k=\overline{0,4}$ 


$$
x_{35 n+16+k}-x_{-14+k}=\left(x_{16+k}-x_{-14+k}\right) \sum_{j=0}^{n} \prod_{i=1}^{6 j+3} \frac{1}{1+x_{5 i-4+k} x_{5 i-9+k} x_{5 i-14+k} x_{5 i-19+k} x_{5 i-24+k}} .
$$

Also, $6 j+4$ inserted in 2.1 by replacing $n, j=0$ to $j=n$ is obtained by summing, for $k=\overline{0,4}$

$$
x_{30 n+21+k}-x_{-9+k}=\left(x_{21+k}-x_{-9+k}\right) \sum_{j=0}^{n} \prod_{i=1}^{6 j+4} \frac{1}{1+x_{5 i-4+k} x_{5 i-9+k} x_{5 i-14+k} x_{5 i-19+k} x_{5 i-24+k}} .
$$

Also, $6 j+5$ inserted in 2.1 by replacing $n, j=0$ to $j=n$ is obtained by summing, for $k=\overline{0,4}$

$$
x_{30 n+26+k}-x_{-4+k}=\left(x_{26+k}-x_{-4+k}\right) \sum_{j=0}^{n} \prod_{i=1}^{6 j+5} \frac{1}{1+x_{5 i-4+k} x_{5 i-9+k} x_{5 i-14+k} x_{5 i-19+k} x_{5 i-24+k}} .
$$

Now we obtained of the above formulas:

$$
\begin{aligned}
& x_{30 n+1+k}=x_{-29+k}\left(1-\frac{x_{-4+k} x_{-9+k} x_{-14+k} x_{-19+k} x_{-24+k}}{x_{-4+k} x_{-9+k} x_{-14+k} x_{-19+k} x_{-24+k}} \sum_{j=0}^{6 j} \frac{1}{1+x_{5 i-4+k} x_{5 i-9+k} x_{5 i-14+k} x_{5 i-19+k} x_{5 i-24+k}}\right), \\
& x_{30 n+6+k}=x_{-24+k}\left(1-\frac{x_{-4+k} x_{-9+k} x_{-14+k} x_{-19+k} x_{-29+k}}{x_{-4+k} x_{-9+k} x_{-14+k} x_{-19+k} x_{-24+k}} \sum_{j=0}^{6 j+1} \frac{1}{1+x_{5 i-4+k} x_{5 i-9+k} x_{5 i-14+k} x_{5 i-19+k} x_{5 i-24+k}}\right) \\
& x_{30 n+11+k}=x_{-19+k}\left(1-\frac{x_{-4+k} x_{-9+k} x_{-14+k} x_{-24+k} x_{-29+k}}{x_{-4+k} x_{-9+k} x_{-14+k} x_{-19+k} x_{-24+k}} \sum_{j=0}^{6 j+2} \frac{1}{1+x_{5 i-4+k} x_{5 i-9+k} x_{5 i-14+k} x_{5 i-19+k} x_{5 i-24+k}}\right) \\
& x_{30 n+16+k}=x_{-14+k}\left(1-\frac{x_{-4+k} x_{-9+k} x_{-19+k} x_{-24+k} x_{-29+k}}{x_{-4+k} x_{-9+k} x_{-14+k} x_{-19+k} x_{-24+k}} \sum_{j=0}^{6 j+3} \frac{1}{1+x_{5 i-4+k} x_{5 i-9+k} x_{5 i-14+k} x_{5 i-19+k} x_{5 i-24+k}}\right) \\
& x_{30 n+21+k}=x_{-9+k}\left(1-\frac{x_{-4+k} x_{-14+k} x_{-19+k} x_{-24+k} x_{-29+k}}{x_{-4+k} x_{-9+k} x_{-14+k} x_{-19+k} x_{-24+k}} \sum_{j=0}^{n} \prod_{i=1}^{6 j+4} \frac{1}{1+x_{5 i-4+k} x_{5 i-9+k} x_{5 i-14+k} x_{5 i-19+k} x_{5 i-24+k}}\right) \\
& x_{30 n+26+k}=x_{-4+k}\left(1-\frac{x_{-9+k} x_{-14+k} x_{-19+k} x_{-24+k} x_{-29+k}}{x_{-4+k} x_{-9+k} x_{-14+k} x_{-19+k} x_{-24+k}} \sum_{j=0}^{n} \prod_{i=1}^{6 j+5} \frac{1}{1+x_{5 i-4+k} x_{5 i-9+k} x_{5 i-14+k} x_{5 i-19+k} x_{5 i-24+k}}\right) \\
& k=\overline{0,4} \text { holds. }
\end{aligned}
$$


e) Suppose that $a_{1}=a_{6}=a_{11}=a_{16}=a_{21}=a_{26}=0$. By (d), the following formulas are produced below

$$
\begin{aligned}
& \lim _{n \rightarrow \infty} x_{30 n+1}=\lim _{n \rightarrow \infty} x_{-29}\left(1-\frac{x_{-4} x_{-9} x_{-14} x_{-19} x_{-24}}{1+x_{-4} x_{-9} x_{-14} x_{-19} x_{-24}} \sum_{j=0}^{n} \prod_{i=1}^{6 j} \frac{1}{1+x_{5 i-4} x_{5 i-9} x_{5 i-14} x_{5 i-19} x_{5 i-24}}\right) \\
& a_{1}=x_{-29}\left(1-\frac{x_{-4} x_{-9} x_{-14} x_{-19} x_{-24}}{1+x_{-4} x_{-9} x_{-14} x_{-19} x_{-24}} \sum_{j=0}^{\infty} \prod_{i=1}^{6 j} \frac{1}{1+x_{5 i-4} x_{5 i-9} x_{5 i-14} x_{5 i-19} x_{5 i-24} x_{5 i-29}}\right) \\
& a_{1}=0 \Rightarrow \frac{1+x_{-4} x_{-9} x_{-14} x_{-19} x_{-24}}{x_{-4} x_{-9} x_{-14} x_{-19} x_{-24}}=\sum_{j=0}^{\infty} \prod_{i=1}^{6 j} \frac{1}{x_{5 i-4} x_{5 i-9} x_{5 i-14} x_{5 i-19} x_{5 i-24}}
\end{aligned}
$$

Similarly,

$$
a_{6}=0 \Rightarrow \frac{1+x_{-4} x_{-9} x_{-14} x_{-19} x_{-24}}{x_{-4} x_{-9} x_{-14} x_{-19} x_{-29}}=\sum_{j=0}^{\infty} \prod_{i=1}^{6 j+1} \frac{1}{x_{5 i-4} x_{5 i-9} x_{5 i-14} x_{5 i-19} x_{5 i-24}}
$$

Similarly,

$$
a_{11}=0 \Rightarrow \frac{1+x_{-4} x_{-9} x_{-14} x_{-19} x_{-24}}{x_{-4} x_{-9} x_{-14} x_{-24} x_{-29}}=\sum_{j=0}^{\infty} \prod_{i=1}^{6 j+2} \frac{1}{x_{5 i-4} x_{5 i-9} x_{5 i-14} x_{5 i-19} x_{5 i-24}} .
$$

Similarly,

$$
a_{16}=0 \Rightarrow \frac{1+x_{-4} x_{-9} x_{-14} x_{-19} x_{-24}}{x_{-4} x_{-9} x_{-19} x_{-24} x_{-29}}=\sum_{j=0}^{\infty} \prod_{i=1}^{6 j+3} \frac{1}{x_{5 i-4} x_{5 i-9} x_{5 i-14} x_{5 i-19} x_{5 i-24}} .
$$

Similarly,

$$
a_{21}=0 \Rightarrow \frac{1+x_{-4} x_{-9} x_{-14} x_{-19} x_{-24}}{x_{-4} x_{-14} x_{-19} x_{-24} x_{-29}}=\sum_{j=0}^{\infty} \prod_{i=1}^{6 j+4} \frac{1}{x_{5 i-4} x_{5 i-9} x_{5 i-14} x_{5 i-19} x_{5 i-24}}
$$

Similarly,

$$
a_{26}=0 \Rightarrow \frac{1+x_{-4} x_{-9} x_{-14} x_{-19} x_{-24}}{x_{-9} x_{-14} x_{-19} x_{-24} x_{-29}}=\sum_{j=0}^{\infty} \prod_{i=1}^{6 j+5} \frac{1}{x_{5 i-4} x_{5 i-9} x_{5 i-14} x_{5 i-19} x_{5 i-24}} .
$$

From 2.2 and 2.3

$$
\begin{aligned}
\frac{1+x_{-4} x_{-9} x_{-14} x_{-19} x_{-24}}{x_{-4} x_{-9} x_{-14} x_{-19} x_{-24}}=\sum_{j=0}^{\infty} \prod_{i=1}^{6 j} \frac{1}{x_{5 i-4} x_{5 i-9} x_{5 i-14} x_{5 i-19} x_{5 i-24}}> & \frac{1+x_{-4} x_{-9} x_{-14} x_{-19} x_{-24}}{x_{-4} x_{-9} x_{-14} x_{-19} x_{-29}}=\sum_{j=0}^{\infty} \prod_{i=1}^{6 j+1} \frac{1}{x_{5 i-4} x_{5 i-9} x_{5 i-14} x_{5 i-19} x_{5 i-24}}
\end{aligned}
$$

thus, $x_{-29}>x_{-24}$. From 2.3 and 2.4 


$$
\begin{aligned}
\frac{1+x_{-4} x_{-9} x_{-14} x_{-19} x_{-24}}{x_{-4} x_{-9} x_{-14} x_{-19} x_{-29}}=\sum_{j=0}^{\infty} \prod_{i=1}^{6 j+1} \frac{1}{x_{5 i-4} x_{5 i-9} x_{5 i-14} x_{5 i-19} x_{5 i-24}}> & \frac{1+x_{-4} x_{-9} x_{-14} x_{-19} x_{-24}}{x_{-4} x_{-9} x_{-14} x_{-24} x_{-29}}=\sum_{j=0}^{\infty} \prod_{i=1}^{6 j+2} \frac{1}{x_{5 i-4} x_{5 i-9} x_{5 i-14} x_{5 i-19} x_{5 i-24}}
\end{aligned}
$$

thus, $x_{-24}>x_{-19}$. From 2.4 and 2.5

$$
\begin{aligned}
\frac{1+x_{-4} x_{-9} x_{-14} x_{-19} x_{-24}}{x_{-4} x_{-9} x_{-14} x_{-24} x_{-29}}=\sum_{j=0}^{\infty} \prod_{i=1}^{6 j+2} \frac{1}{x_{5 i-4} x_{5 i-9} x_{5 i-14} x_{5 i-19} x_{5 i-24}}> & \frac{1+x_{-4} x_{-9} x_{-14} x_{-19} x_{-24}}{x_{-4} x_{-9} x_{-19} x_{-24} x_{-29}}=\sum_{j=0}^{\infty} \prod_{i=1}^{6 j+3} \frac{1}{x_{5 i-4} x_{5 i-9} x_{5 i-14} x_{5 i-19} x_{5 i-24}}
\end{aligned}
$$

thus, $x_{-19}>x_{-14}$. From 2.5 and 2.6

$$
\begin{aligned}
\frac{1+x_{-4} x_{-9} x_{-14} x_{-19} x_{-24}}{x_{-4} x_{-9} x_{-19} x_{-24} x_{-29}}=\sum_{j=0}^{\infty} \prod_{i=1}^{6 j+3} \frac{1}{x_{5 i-4} x_{5 i-9} x_{5 i-14} x_{5 i-19} x_{5 i-24}}> & \frac{1+x_{-4} x_{-9} x_{-14} x_{-19} x_{-24}}{x_{-4} x_{-14} x_{-19} x_{-24} x_{-29}}=\sum_{j=0}^{\infty} \prod_{i=1}^{6 j+4} \frac{1}{x_{5 i-4} x_{5 i-9} x_{5 i-14} x_{5 i-19} x_{5 i-24}}
\end{aligned}
$$

thus, $x_{-14}>x_{-9}$. From 2.6 and 2.7

$$
\begin{aligned}
\frac{1+x_{-4} x_{-9} x_{-14} x_{-19} x_{-24}}{x_{-4} x_{-14} x_{-19} x_{-24} x_{-29}}=\sum_{j=0}^{\infty} \prod_{i=1}^{6 j+4} \frac{1}{x_{5 i-4} x_{5 i-9} x_{5 i-14} x_{5 i-19} x_{5 i-24}}> & \frac{1+x_{-4} x_{-9} x_{-14} x_{-19} x_{-24}}{x_{-9} x_{-14} x_{-19} x_{-24} x_{-29}}=\sum_{j=0}^{\infty} \prod_{i=1}^{6 j+5} \frac{1}{x_{5 i-4} x_{5 i-9} x_{5 i-14} x_{5 i-19} x_{5 i-24}}
\end{aligned}
$$

thus, $x_{-9}>x_{-4}$.

From here we obtain $x_{-29}>x_{-24}>x_{-19}>x_{-14}>x_{-9}>x_{-4}$. Similarly, we can obtain $x_{-28}>x_{-23}>x_{-18}>x_{-13}>$ $x_{-8}>x_{-3}, x_{-27}>x_{-22}>x_{-17}>x_{-12}>x_{-7}>x_{-2}, x_{-26}>x_{-21}>x_{-16}>x_{-11}>x_{-6}>x_{-1}$ and $x_{-25}>x_{-20}>x_{-15}>$ $x_{-10}>x_{-5}>x_{0}$. We arrive at a contradiction which completes the proof of theorem.

\section{Conclusion}

In this study, the theorem is given for the 1.1, and its solution and periodicity are investigated. By taking the coefficients of the 1.1 , real numbers, sequence or function, new equations can be defined and their solutions can be examined.

\section{References}

[1] A.M. Amleh, G.A. Grove, G. Ladas, D.A. Georgiou, On the recursive sequence $y_{n+1}=\alpha+\frac{y_{n-1}}{y_{n}}$ J. of Math. Anal. App., 233, (1999), 790-798.

[2] C. Cinar, On the positive solutions of the difference equation $x_{n+1}=\frac{x_{n-1}}{-1+\alpha x_{n} x_{n-1}}$, J. of App. Math. Comp., 158(3), (2004), 793-797.

[3] C. Cinar, T. Mansour, I. Yalcinkaya, On the difference equation of higher order, Utilitas Mathematica, 92, (2013), 161-166. 
On the Recursive Sequence $x_{n+1}=\frac{x_{n-29}}{1+x_{n-4} x_{n-9} x_{n-14} x_{n-19} x_{n-24}}-\mathbf{5 3 / 5 4}$

[4] C.H. Gibbons, M.R.S. Kulenovic, G. Ladas, On the recursive sequence $\frac{\alpha+\beta x n-1}{\chi+\beta x n-1}$, Math. Sci. Res. Hot-Line, 4(2), (2000), 1-11.

[5] D. Simsek, C. Cinar, I. Yalcinkaya, On the recursive sequence $x_{n+1}=\frac{x_{n-3}}{1+x_{n-1}}$, Int J. Contemp., 9(12), (2006), 475-480.

[6] D. Simsek, C. Cinar, I. Yalcinkaya, On the recursive sequence $x_{n+1}=\frac{x_{n-5}}{1+x_{n-2}}$, Int J. Pure Appl. Math., 27, (2006), 501-507.

[7] D. Simsek, C. Cinar, I. Yalcinkaya, On the recursive sequence $x_{n+1}=\frac{x_{n-5}}{1+x_{n-1} x_{n-3}}$, Int J. Pure Appl. Math., 28, (2006), 117-124.

[8] D. Simşek, B. Ogul, C. Cinar, Solution of the rational difference equation $x_{n+1}=\frac{x_{n-17}}{1+x_{n-5} x_{n-11}}$, Filomat, 33(5), (2019), 1353-1359.

[9] D. Simsek, B. Ogul, F. Abdullayev, Solution of the Rational Difference Equation $x_{n+1}=\frac{x_{n-13}}{1+x_{n-1} x_{n-3} x_{n-5} x_{n-7} x_{n-9} x_{n-11}}$, Applied Mathematics and Nonlinear Sciences, 5(1), (2020), 485-494.

[10] E.M. Elsayed, On the solution of some difference equation, Europan Journal of Pure and Applied Mathematics, 4(3), (2011), 287-303.

[11] E.M. Elsayed, On the Dynamics of a higher order rational recursive sequence, Communications in Mathematical Analysis, 12(1), (2012), 117-133.

[12] E.M. Elsayed, Solution of rational difference system of order two, Mathematical and Computer Modelling, 5, (2012), 378-384.

[13] E.M. Elsayed, Behavior and expression of the solutions of some rational difference equations, Journal of Computational Analysis Applications, 15(1), (2013), 73-81.

[14] E.M. Elsayed, Solution of rational difference system of order two, Journal of Computational Analysis Applications, 33(3), (2014), 751-765.

[15] E.M. Elsayed, Solution and attractivity for a rational recursive sequence, Discrete Dynamics in Nature and Society, 2011, (2011), 1-17.

[16] E.M. Elabbasy, H. El-Metwally, E.M. Elsayed, On the difference equation $x_{n+1}=\frac{\alpha_{x} n_{k}}{\beta}+\gamma \pi=0$, Journal of Concrete Applicable Mathematics, 5(2), (2007), 101-103.

[17] E.M. Erdogan, C. Cinar, I. Yalcinkaya, On the dynamics of the recursive sequence, Mathematical and Computer Modelling, 54(5), (2011), 1481-1485.

[18] F. Belhannache, N. Touafek, R. Abo-Zeid, On a higher-order rational difference equation J. Appl. Math. Informatics, 34(5-6), (2016), 369-382.

[19] M. Lui, Z. Guo, Solvability of a higher-order nonlinear neutral delay difference equation, Advances in Difference Equations, 2010(1), (2010), 620-627.

[20] M. Ari, A. Gelisken, Periodic and asymptotic behavior of a difference equation, Asian-European Journal of Mathematics, 12(6), (2019), 2040004.

[21] M.R.S. Kulenovic, G. Ladas, W.S. Sizer, On the recursive sequence $\frac{\alpha x_{n}+\beta x_{n-1}}{\chi x_{n}+\beta x_{n-1}}$, Math. Sci. Res.Hot-Line, 2(5), (1998), 1-16.

[22] O. Moaaz, D. Chalishajar, O. Bazighifan, Some qualitative behavior of solutions of general class of difference equations. Mathematics, 7(7), (2019), 585.

${ }^{[23]}$ R. DeVault, G. Ladas, S.W. Schultz, On the recursive sequence $x_{n+1}=\frac{A}{x_{n}}+\frac{1}{x_{n-2}}$, Proc.Amer. Math. Soc., 126(11), (1998), 3257-3261.

[24] R.P. Agarwal, E.M. Elsayed, On the solution of fourth-order rational recursive sequence, Advanced Studies in Contemporary Mathematics, 20(4),(2010), 525-545.

[25] R. Karatas, C. Cinar, D. Simsek, On Positive Solutions of the Difference Equation $x_{n+1}=\frac{x_{n-5}}{1+x_{n-2} x_{n-5}}$, Int. J. Contemp. Math. Sci, 10(1), (2006), 495-500. 
On the Recursive Sequence $x_{n+1}=\frac{x_{n-29}}{1+x_{n-4} x_{n-9} x_{n-14} x_{n-19} x_{n-24}}-\mathbf{5 4 / 5 4}$

[26] R. Karatas, Global behavior of a higher order difference equation, Int. J. Contemp. Math. Sci., 12(3), (2017), 133-138.

[27] S.E. Das, M. Bayram, On a system of rational difference equations, World Applied Sciences Journal, 10(11), (2010), 1306-1312.

[28] S. Stevic, B. Iricanin, Z. Smarda, On a product-type system of difference equations of second order solvable in closed form, Journal of Inequalities and Applications, 2015(1), (2012), 327-334.

[29] T.F. Ibrahim, Behavior of some higher order nonlinear rational partial difference equations, Journal of the Egyptian Mathematical Society, 24(4), (2016), 532-537.

[30] T.F. Ibrahim, Bifurcation and periodically semicycles for fractional difference equation of fifth order Journal of Nonlinear Sciences and Applications, 11(3), (2018), 375-382. 